\title{
WT1 overexpression affecting clinical outcome in non-Hodgkin lymphomas and adult acute lymphoblastic leukemia
}

\author{
Zsófia UJJ, ${ }^{1 *}$ Gergely BUGLYÓ, ${ }^{2 *}$ Miklós UDVARDY, ${ }^{1}$ György VARGHA, ${ }^{2}$ Sándor BIRÓ, ${ }^{2}$ László \\ REJTÖ $^{1}$
}

${ }^{1}$ Institute of Internal Medicine, Division of Haematology, ${ }^{2}$ Department of Human Genetics, Medical and Health Science Center, University of Debrecen, Debrecen, Hungary

*These authors contributed equally to this work.

Correspondence: Gergely Buglyó, Department of Human Genetics, Medical and Health Science Center, University of Debrecen, 4032 Debrecen, Nagyerdei krt. 98, Hungary. Tel.: +36-52/416-531 Fax: +3652/416-531 E-mail: gbuglyo@ hotmail.com

\begin{abstract}
The Wilms tumor 1 (WT1) gene has a complex role as a transcriptional regulator, acting as tumor suppressor or oncogene in different malignancies. The prognostic role of its overexpression has been well-studied in leukemias, especially acute myeloid leukemia (AML), but not in lymphomas. For the first time to our knowledge, we present a study demonstrating the correlation of WT1 expression and survival in various non-Hodgkin lymphomas. We also studied the prognostic implications of WT1 overexpression in adult acute lymphoblastic leukemia (ALL). In our sample of 53 patients - 25 with diffuse large B-cell lymphoma (DLBCL), 8 with mantle cell lymphoma (MCL), 9 with peripheral T-cell lymphoma (PTCL), 2 with Burkitt's lymphoma, 2 with mucosa-associated lymphoid tissue (MALT) lymphoma, and 7 with B-cell ALL -, we measured WT1 mRNA from blood samples by quantitative RTPCR, and divided the patients into subgroups based on the level of expression. Kaplan-Meier survival curves were drawn and compared using the logrank test. In the sample of DLBCL patients, the difference in overall and disease-free survival between WT1-positive and negative subgroups was significant ( $\mathrm{p}=0,0475$ and $\mathrm{p}=0,0004$, respectively), and in a few observed cases, a sudden increase in WT1 expression signified a relapse soon followed by death. Disease-free survival curves in MCL and ALL were similarly suggestive of a potential role played by WT1. In PTCL, though WT1-positivity was detected in 4 out of 9 cases, it did not seem to affect survival. The few cases of MALT and Burkitt's lymphoma all proved to be WT1-negative.
\end{abstract}

Keywords: WT1 gene, overexpression, non-Hodgkin lymphoma, diffuse large B-cell lymphoma, mantle cell lymphoma, acute lymphoblastic leukemia

\section{Introduction}

The Wilms tumor 1 (WT1) gene is located at chromosomal region 11p13. It encodes a DNA-binding protein with 4 zinc finger domains, which has long been known to act as a tumor suppressor in Wilms tumor [1]. In other neoplasms, however, WT1 seems to play the opposite role as an oncogene. This may be due to a two-sided function of WT1 promoting differentiation of cells in the genitourinary tract, while maintaining an immature mesenchymal state in other tissues [2]. Complexity of the protein's function is mainly attributed to the large number of isoforms: the first 4 were characterized in 1991 [3], but other variants were soon discovered, reaching a total of 24 identified isoproteins by the late $90 \mathrm{~s}-$ and the theoretical limit of possible isoforms has only grown since then, as further data accumulated [4]. Still, the 2 isoforms either including or excluding a tripeptide (KTS) due to the alternative splicing of exon 9 seem to be the most important from a practical aspect [5]. Apart from DNA-binding, WT1 might have a regulatory role at the posttranscriptional level as well [6].

Reports on high levels of WT1 expression in acute leukemias were published only 2 years after the gene's discovery [7], and WT1-positive AML cases were associated with a bad prognosis [8]. Recently, WT1 siRNA was used in the treatment of leukemic cell lines with some success [9], but the oncogenic role of 
WT1 in hematological malignancies remains poorly understood. Besides the assessment of prognosis at diagnosis, measuring WT1 expression is also considered suitable for monitoring minimal residual disease in AML $[10,11]$.

WT1 expression has been extensively studied in AML and myelodysplastic syndrome (MDS). In recent years, some results have been reported in childhood and adult ALL as well. These suggest that while WT1 expression is not a reliable marker of prognosis and minimal residual disease in ALL, WT1 could still become a therapeutic target, as its overexpression was detected in a sufficiently high number of cases [12-14]. For the majority of lymphomas, however, no studies have been published to report the possible prognostic significance of WT1 expression, even though a putative role of WT1 in their pathogenesis has been suggested, much like in acute leukemias [15].

Here, we studied WT1 expression in patients with different non-Hodgkin lymphomas and ALL, and examined the correlation between detected expression levels and clinical outcome.

\section{Materials and Methods}

53 patients were studied between March 2007 and May 2012: 25 with DLBCL, 8 with MCL, 9 with PTCL, 2 with Burkitt's lymphoma, 2 with MALT lymphoma, and 7 with B-cell ALL. Morphological and immunophenotypic (by flow cytometry) classification was used to confirm cases of ALL, while in nonHodgkin lymphomas, a surgical lymph node excision or biopsy was used as a tissue source for pathological diagnosis, and the Ann Arbor system was applied for clinical staging. We took blood samples (multiple times in most cases: at diagnosis, during and after treatment) until the end of the study, last follow-up, or death of the patient. Written informed consent was obtained from all patients in accordance with the Declaration of Helsinki.

Samples were collected in PAXgene Blood RNA Tubes, and stored at $-20{ }^{\circ} \mathrm{C}$. We used PAXgene Blood RNA Kits to extract RNA, and after reverse transcription by High Capacity cDNA Reverse Transcription Kits (Applied Biosystems), we detected cDNA levels using an Applied Biosystems 7500 Real Time PCR System with primers and a fluorescent Taqman probe specific to WT1 (commercial kit No. Hs00240913_m1) and a reference gene showing high and relatively constant expression (GAPDH). The housekeeping gene GAPDH is still favored as endogenous control in the literature, since its expression shows no difference in normal and neoplastic lymphoid tissue [16]. Rather than calculating with $\mathrm{Ct}$ values, we normalized WT1 expression against GAPDH, and used the resulting value (number of WT1 mRNA molecules per $10^{4}$ GAPDH mRNA molecules) for further calculations. This method eliminated the problem of RNA extraction with varying degrees of efficiency from different samples, as we produced reliable results at low RNA concentrations, even below $1 \mathrm{ng} / \mu \mathrm{L}$ in a few cases (most of our RNA samples were in the $200-500 \mathrm{ng} / \mu \mathrm{L}$ range).

We performed statistical analysis on calculated WT1 expression values using the software MedCalc (version 12.3.0). Overall survival was calculated from the date of diagnosis until death or last follow-up. (Some of the patients were diagnosed earlier, and had been under treatment for years when they entered our study.) Disease-free survival was defined as the time spent in remission until relapse, death or last follow-up. Figures in our paper were also drawn by MedCalc.

\section{Controls}

Our control population consisted of 35 healthy individuals of different age and sex, who volunteered to provide blood samples at certain times through the study. WT1 expression values (normalized against GAPDH) from the 35 samples ranged from 0,002 to 0,109 . We performed a chi-square goodness-of-fit test for normal distribution on control values (including a logarithmic transformation of data). According to the result, normality could be accepted $(\mathrm{p}=0,9923)$. We took the value at 97,5 percentile $(0,108)$, and rounded it to 0,1 for easier use as a cut-off value for WT1-positivity in our study. 


\section{Results}

\section{Diffuse large B-cell lymphoma}

Out of 53 patients, 25 were presented with DLBCL, so this group represents our largest sample. Median age at diagnosis was 54 years, ranging from 24 to 77 years. Treatment included CHOP and DHAP chemotherapy protocols combined with rituximab (monoclonal antibody against the CD20 antigen), and irradiation, autologous stem cell transplantation in certain cases.

WT1-positivity was detected in 8 out of 25 cases, and Kaplan-Meier survival curves were drawn to study its effects on clinical outcome (Fig. 1). In overall survival, the WT1-positive subgroup produced a steeper curve - the observation was confirmed by a logrank test $(\mathrm{p}=0,0475)$. The difference in clinical outcome was even more striking when studying disease-free survival $(\mathrm{p}=0,0004)$. In 2 patients, we experienced an elevation over the cut-off value in a previously negative WT1 expression pattern. This seems to have been a sign of relapse: in one patient, WT1 expression was 0,002 during a partial remission, but increased to 0,139 as measured in a sample taken on the day of her death due to a relapse, while in another case, a previously low expression value $(0,0019)$ showed a massive peak of 226,0 during a relapse, 6 weeks preceding death.
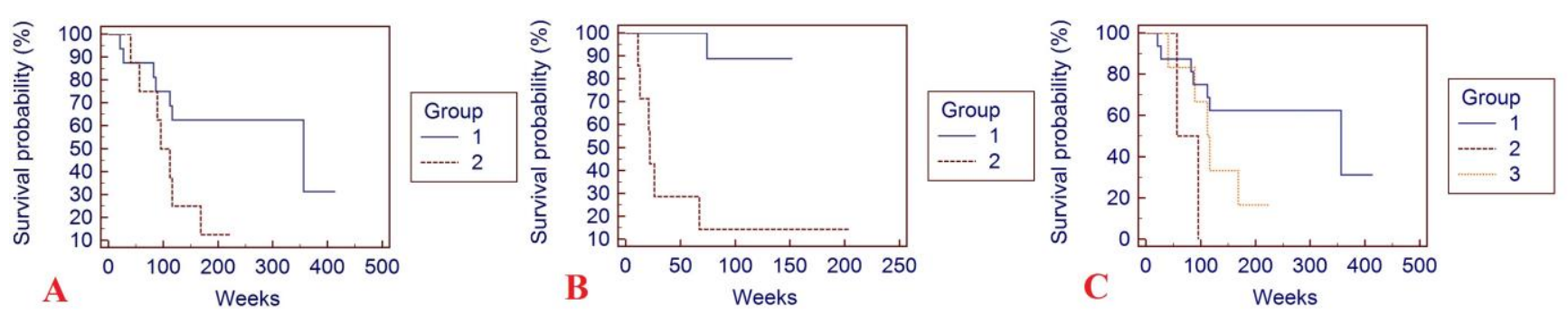

Fig. 1 Kaplan-Meier curves representing survival of DLBCL patients. Overall survival (A) and diseasefree survival (B) were studied in WT1-negative (Group 1) and WT1-positive (Group 2) patients. In diagram $\mathrm{C}$, we illustrated the difference in overall survival between patients who were WT1-negative throughout the study (Group 1), patients who started out negative but became positive at some point (Group 2), and patients who presented only WT1-positive samples (Group 3)

\section{Mantle cell lymphoma}

We had 8 cases of MCL, with an age range of 38 to 74 years at diagnosis (median age was 60 years). Hyper-CVAD, CHOP, DHAP and BEAM (the latter followed by autologous stem cell transplantation) protocols were used for treatment, most often administered together with rituximab. WT1-positivity was detected in 4 cases. One of the patients (pretreated for MCL 5 years before) expressed a value of 0,0026 when entering our study, but presented an unusually high level of expression $(2,07)$ a year later. Since his bone marrow sample showed signs of MDS, and he died of MDS 18 months afterwards, it seemed reasonable to omit the case from the MCL statistics and report it separately.

Overall and disease-free survival was compared in WT1-positive and negative subgroups among the remaining 7 patients (Fig. 2). Kaplan-Meier curves and the logrank test seem to suggest a longer overall $(\mathrm{p}=0,0082)$ and disease-free survival $(\mathrm{p}=0,0177)$ for WT1-negative patients. Changes in WT1 expression through the observation period may also be worth noting: a patient who showed a positive value of 0,288 at diagnosis went negative $(0,0018)$ after 4 months of hyper-CVAD treatment, and he survived more than 2 years until the last follow-up. 

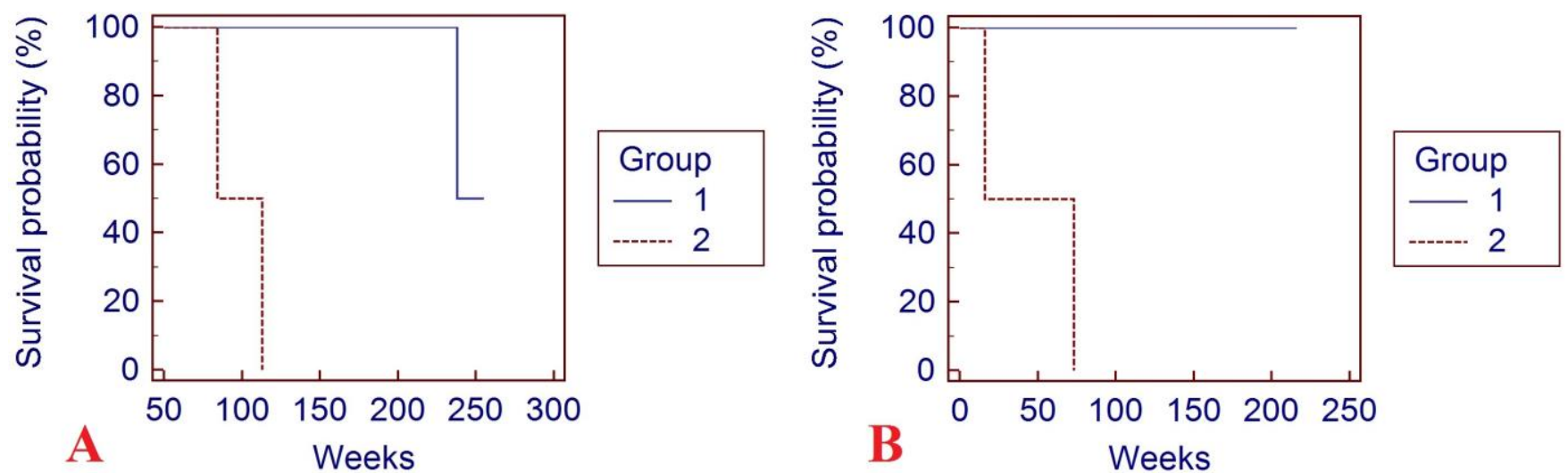

Fig. 2 Overall (A) and disease-free (B) survival in 7 MCL patients. Group 1 contained 4 patients who were WT1-negative over the course of our study, and 1 patient who was positive at diagnosis, but decreased below the cut-off value through therapy. Group 2 contained 2 WT1-positive patients (one of them showed negative values in the beginning, but became positive during the observation period)

\section{Peripheral T-cell lymphoma}

We had a group of 9 patients presented with PTCL at a median age of 50 years (range: 32 to 67 years). Classic chemotherapy protocols (CHOP, DHAP, BEAM) were applied, sometimes in combination with alemtuzumab. Autologous stem cell transplantation was also used in cases where (at least) partial remission had been achieved.

WT1 expression over the cut-off value for positivity $(0,1)$ was detected in 4 cases. However, unlike B-cell lymphomas detailed above, survival curves in PTCL showed no difference between WT1-positive and negative subgroups (Fig. 3) - results of logrank tests were $\mathrm{p}=0,3198$ for overall, and $\mathrm{p}=0,8084$ for disease-free survival.
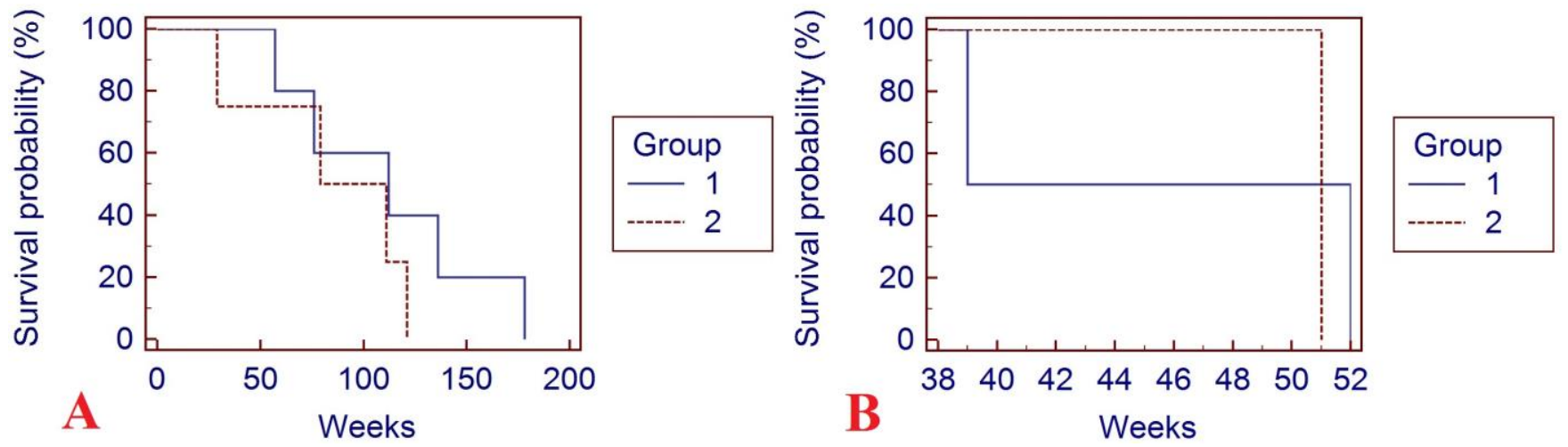

Fig. 3 Kaplan-Meier survival curves in PTCL: group 1 consisted of WT1-negative, and group 2 of WT1positive cases. Overall survival is shown in diagram A, and disease-free survival in diagram B

\section{Adult acute lymphoblastic leukemia}

We examined a smaller group of 7 patients with B-cell ALL. Age at diagnosis ranged between 19 and 65 years, with a median of 48 years. Treatment consisted of Hoelzer regimen [17] with Glivec in cases where translocation $\mathrm{t}(9 ; 22)$ was detected.

WT1-positivity was detected in 5 cases, and its effect on survival was examined (Fig. 4). 3 cases that were included in overall survival analysis died within 7 weeks after diagnosis, and 2 of them were WT1negative, so Kaplan-Meier curves were unremarkable. On the other hand, disease-free survival could be studied in 4 patients who all presented elevated WT1 expression in their first sample at diagnosis. Two of them remained positive, and died in relapses 25 and 41 weeks after reaching a temporary remission, while 2 became negative after induction treatment. Clinically, they have remained in remission since then. A 
logrank test comparing Kaplan-Meier curves for disease-free survival is not far from being significant $(\mathrm{p}=0,0896)$, which may be worth noting, given the small sample size.
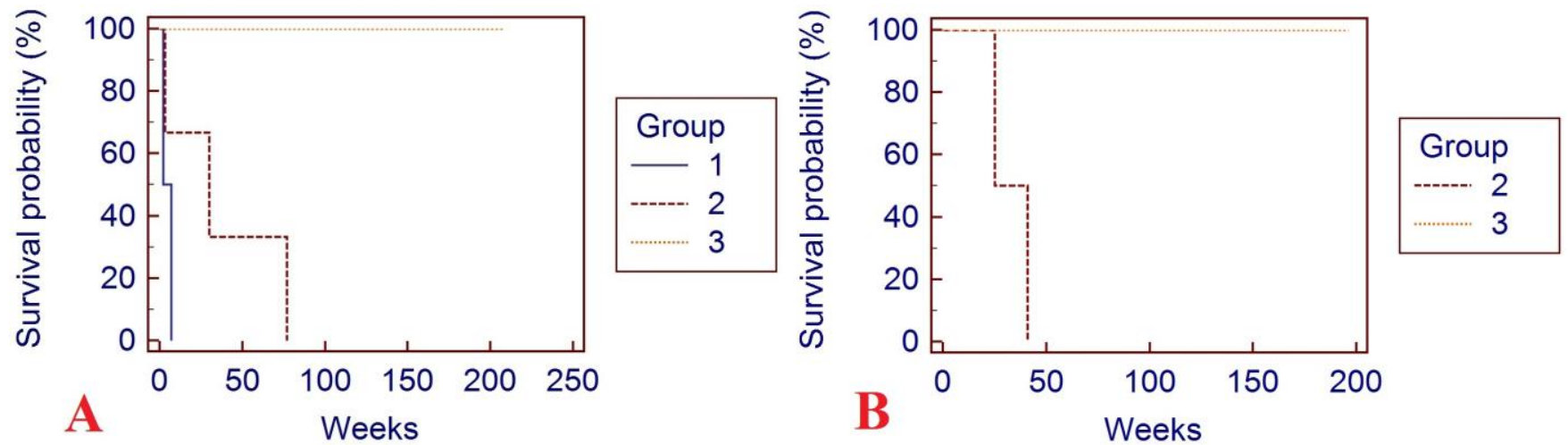

Fig. 4 Effects of WT1 overexpression on survival in adult ALL patients: overall (A) and disease-free (B) survival. Group 1 contained 2 WT1-negative patients (they died 2 and 7 weeks after diagnosis, so diseasefree survival is not applicable). Group 2 consisted of 3 patients whose WT1 expression remained elevated through the course of our study, while group 3 had 2 patients who showed high WT1 expression at diagnosis that soon decreased into normal range during treatment

\section{MALT and Burkitt's lymphoma}

We had 2 patients with MALT, and 2 with Burkitt's lymphoma. All 4 cases proved to be WT1-negative. Remission was not achieved in either case of Burkitt's lymphoma, and both patients died (after 18 and 38 weeks, respectively). On the other hand, MALT lymphoma cases responded well to treatment, and both patients remain in remission after 203 and 136 weeks, respectively.

\section{Discussion}

The few studies available in the literature at present on the role of WT1 in lymphomas have all used immunohistochemical methods to detect the gene's overexpression in tumor samples from various nonHodgkin lymphomas [15,18], and malignant lymph nodes from Hodgkin patients [19]. The latter study suggested WT1 as a possible proangiogenic factor in Hodgkin lymphoma. Wang et al. reported that detected levels of WT1 expression in anaplastic large-cell lymphoma (ALCL) did not have a significant impact on prognosis [18], but interpretation of their results is limited by the method of analyzing tumor samples taken at diagnosis, which only allows calculation of overall survival. Based on our own results and earlier studies on acute leukemias [11], we cannot exclude the possibility that the conclusion might have been different if they had determined disease-free survival as well. When planning our study, the first difficult decision was what type of sample and method to use.

One could argue that samples from lymph nodes or tumor tissue are better to detect WT1 from, as they contain a much higher concentration of neoplastic cells than blood does. Our aim, however, was to obtain a relatively large number of samples over a certain time interval from each patient, and use qRT-PCR for fast analysis, in order to determine a correlation between the molecular and clinical state of the disease, and evaluate WT1 as a potential prognostic factor. One problem with neoplastic lymph node and tumor tissue samples is that they are no longer available after surgical removal. The other problem is that control samples are hard to come by, so it is not easy to decide what level of expression should be considered "WT1-positive". Comparison to values in literature does not help, either, since procedures of collecting and storing samples as well as analyzing them in the laboratory can be so different that results are unlikely to be on the same scale. While measuring WT1 expression is not easy even by qRT-PCR, we believe that our method of using blood samples (with a fairly large pool of controls) and characterizing gene expression by numerical values (after normalization against GAPDH) has produced a reliable cut-off value that could be used as a threshold for WT1-positivity in our study. 
In DLBCL, our results suggest that WT1 might be a useful marker to assess prognosis and monitor treatment, although our sample pool was not large, and included patients who were not available for sampling at the time of diagnosis. WT1-positivity seems to be present in about one-third of the cases: 6 out of 18 cases in an earlier study [15], and 8 out of 25 in ours. This observation also implies that the detected frequency of WT1-positivity may not depend on the analyzed sample type (blood or lymph node). WT1-positive patients showed significantly worse overall and disease-free survival under the same treatment protocols. The difference in significance in favor of disease-free survival is to be expected: overall survival analysis included cases at various Ann Arbor stages at diagnosis, so short-term survival did not show much of a difference (Fig. 1A).

To our knowledge, we document cases of WT1-positive MCL for the first time: Drakos et al. reported 6 cases of MCL, none of which expressed WT1 [15]. We think that the difference in survival between WT1-positive and WT1-negative MCL patients is somewhat suggestive for a possible oncogenic role by WT1, although interpretation of the data is difficult due to the small sample size and the diverse range of chemotherapeutics used in different cases as required by international protocols in MCL. We experienced a substantial elevation in WT1 expression, and concurrent signs of MDS in the bone marrow in a patient pretreated for MCL. It is known in the literature that overexpression of WT1 may also be interpreted as an early sign of MDS [20]. In this light, the level of WT1 expression in lymphoma patients takes on a new significance as a marker for MDS or secondary AML in heavily pretreated patients.

Finding both cases of Burkitt's lymphoma to be WT1-negative was contrary to our previous expectations. However, evidence regarding the presence and significance of WT1 overexpression is somewhat controversial in the literature as well $[15,21]$.

Our findings in B-cell ALL fit in with the results of recent large-scale studies [12-14], including the observation that a sudden increase in WT1 expression seems to be associated with an increased risk of relapse. However, our results in ALL represent only a minor contribution to previous reports, since our sample size was relatively small.

Although our own results in T-cell lymphoproliferative disorders are somewhat difficult to interpret, WT1 is hypothesized to play a role in the pathogenesis of certain high-grade T-cell non-Hodgkin lymphomas [15]. Tosello et al. found WT1 mutations in $13.2 \%$ of pediatric and $11.7 \%$ of adult T-ALL, but according to survival analysis, cases with WT1 mutations did not have an adverse prognosis in either pediatric or adult T-ALL [22]. In a recent study, 20 of the 238 T-ALL patients analyzed had WT1 mutations in exon 7. WT1 mutations resulted in an inferior relapse-free survival in standard risk thymic T-ALL patients [23].

We can conclude that apart from its well-known role in acute leukemias, WT1 might also act as an oncogene in certain non-Hodgkin lymphomas. The least we should consider is that WT1 gene expression analysis might provide relevant information on disease progression in DLBCL, providing a tool for the more accurate assessment of remissions and relapses. We propose further investigation - a study should be conducted to evaluate survival in a larger group of DLBCL patients, applying a unified sampling strategy. Based on the results of future studies, WT1 might even become a therapeutic target in cases involving a relapse that resists conventional treatment, as it did in leukemias [24].

\section{Acknowledgement}

This work was supported by the Social Renewal Operational Programme of Hungary.

\section{References}

1. Call KM, Glaser T, Ito CY, Buckler AJ, Pelletier J, Haber DA, Rose EA, Kral A, Yeger H, Lewis WH (1990) Isolation and characterization of a zinc finger polypeptide gene at the human chromosome 11 Wilms' tumor locus. Cell 60(3):509-520 
2. Hohenstein P, Hastie ND (2006) The many facets of the Wilms' tumour gene, WT1. Hum Mol Genet 15:R196-R201

3. Haber DA, Sohn RL, Buckler AJ, Pelletier J, Call KM, Housman DE (1991) Alternative splicing and genomic structure of the Wilms tumor gene WT1. Proc Natl Acad Sci USA 88(21):96189622

4. Dallosso AR, Hancock AL, Brown KW, Williams AC, Jackson S, Malik K (2004) Genomic imprinting at the WT1 gene involves a novel coding transcript (AWT1) that shows deregulation in Wilms' tumours. Hum Mol Genet 13:405-415

5. Hastie ND (2001) Life, sex, and WT1 isoforms - three amino acids can make all the difference. Cell 106:391-394

6. Morrison AA, Viney RL, Ladomery MR (2008) The post-transcriptional roles of WT1, a multifunctional zinc-finger protein. Biochim Biophys Acta 1785:55-62

7. Miwa H, Beran M, Aunders GF (1992) Expression of the Wilms tumor gene (WT1) in human leukemias. Leukemia 6:405-409

8. Bergmann L, Miething C, Maurer U, Brieger J, Karakas T, Weidmann E, Hoelzer D (1997) High levels of Wilms' tumor gene (wt1) mRNA in acute myeloid leukemias are associated with a worse long-term outcome. Blood 90:1217-1225

9. Glienke W, Maute L, Koehl U, Esser R, Milz E, Bergmann L (2007) Effective treatment of leukemic cell lines with wt1 siRNA. Leukemia 21:2164-2170

10. Candoni A, Toffoletti E, Gallina R, Simeone E, Chiozzotto M, Volpetti S, Fanin R (2011) Monitoring of minimal residual disease by quantitative WT1 gene expression following reduced intensity conditioning allogeneic stem cell transplantation in acute myeloid leukemia. Clin Transplant 25(2):308-316

11. Nowakowska-Kopera A, Sacha T, Florek I, Zawada M, Czekalska S, Skotnicki AB (2009) Wilms' tumor gene 1 expression analysis by real-time quantitative polymerase chain reaction for monitoring of minimal residual disease in acute leukemia. Leuk Lymphoma 50(8):1326-1332

12. Boublikova L, Kalinova M, Ryan J, Quinn F, O'Marcaigh A, Smith O, Browne P, Stary J, McCann SR, Trka J, Lawler M (2006) Wilms' tumor gene 1 (WT1) expression in childhood acute lymphoblastic leukemia: a wide range of WT1 expression levels, its impact on prognosis and minimal residual disease monitoring. Leukemia 20(2):254-263

13. Busse A, Gökbuget N, Siehl JM, Hoelzer D, Schwartz S, Rietz A, Thiel E, Keilholz U (2009) Wilms' tumor gene 1 (WT1) expression in subtypes of acute lymphoblastic leukemia (ALL) of adults and impact on clinical outcome. Ann Hematol 88(12):1199-1205

14. Sadek HA, El-Metnawey WH, Shaheen IA, Korshied MM, Mohamed AS (2011) Quantitative assessment of Wilms tumor 1 (WT1) gene transcripts in Egyptian acute lymphoblastic leukemia patients. J Investig Med 59(8):1258-1262

15. Drakos E, Rassidakis GZ, Tsioli P, Lai R, Jones D, Medeiros LJ (2005) Differential expression of WT1 gene product in non-Hodgkin lymphomas. Appl Immunohistochem Mol Morphol 13:132137 
16. Mazrouei S, Ziaei A, Tanhaee AP, Keyhanian K, Esmaeili M, Baradaran A, Salehi M (2012) Apoptosis inhibition or inflammation: the role of NAIP protein expression in Hodgkin and nonHodgkin lymphomas compared to non-neoplastic lymph node. J Inflamm Lond 9:4

17. Hoelzer D, Thiel E, Löffler H, Bodenstein H, Plaumann L, Büchner T, Urbanitz D, Koch P, Heimpel H, Engelhardt R (1983) Recruiting patients and results of a preliminary study on the therapy of acute lymphatic leukemia and acute undifferentiated leukemia in adults. Onkologie 6(4):170-174

18. Wang YF, Yang YL, Gao ZF, Zhou CJ, Gregg X, Shi YF, Wang J, Yang XF, Ke XY (2012) Clinical and laboratory characteristics of systemic anaplastic large cell lymphoma in Chinese patients. J Hematol Oncol 5:38

19. Vadasz Z, Shasha-Lavsky H, Nov Y, Bejar J, Lurie M, Tadmor T, Attias D (2012) Wilms' Tumor Gene 1: A Possible New Proangiogenic Factor in Hodgkin Lymphoma. Appl Immunohistochem Mol Morphol. doi: 10.1097/PAI.0b013e318259852a

20. Gurbuxani, S (2007) WT1 expression in myelodysplastic syndrome. Leuk Lymphoma, 48(3):456457

21. Perotti D, Mondini P, Giardini R, Ferrari A, Massimino M, Gambirasio F, Pierotti MA, FossatiBellani F, Radice P (1998) No evidence of WT1 involvement in a Burkitt's lymphoma in a patient with Denys-Drash syndrome. Ann Oncol 9(6):627-631

22. Tosello V, Mansour MR, Barnes K, Paganin M, Sulis ML, Jenkinson S, Allen CG, Gale RG, Linch DC, Palomero T, Real P, Murty V, Yao X, Richards SM, Goldstone A, Rowe J, Basso G, Wiernik PH, Paietta E, Pieters R, Horstmann M, Meijerink JPP, Ferrando AA (2009) WT1 mutations in T-ALL. Blood 114(5):1038-1045

23. Heesch S, Goekbuget N, Stroux A, Tanchez JO, Schlee C, Burmeister T, Schwartz S, Blau O, Keilholz U, Busse A, Hoelzer D, Thiel E, Hofmann WK, Baldus CD (2010) Prognostic implications of mutations and expression of the Wilms tumor 1 (WT1) gene in adult acute Tlymphoblastic leukemia. Haematologica 95(6):942-949

24. Oka Y, Tsuboi A, Oji Y, Kawase I, Sugiyama H (2008) WT1 peptide vaccine for the treatment of cancer. Curr Opin Immunol 20(2):211-220 